\title{
Separation of variables for Gaudin-Calogero
} systems

\author{
B. ENRIQUEZ ${ }^{1}$, B. FEIGIN ${ }^{2}$ and V. RUBTSOV ${ }^{3}$ \\ ${ }^{1}$ Centre de Mathématiques, U.R.A., 169 du C.N.R.S., Ecole Polytechnique, 91128 Palaiseau, France. \\ ${ }^{2}$ Landau Inst. for Theor. Physics, Kosygina 2, GSP-1, 117940 Moscow V-334, Russia. \\ ${ }^{3}$ ITEP, Bol. Cheremushkinskaya, 25, 117259, Moscow, Russia.
}

Received 30 May 1996; accepted in final form 2 September 1996

\begin{abstract}
We construct an elliptic analogue of Sklyanin's separation of variables for the sl(2) Gaudin system, using an adaptation of Drinfeld's Radon transformations.
\end{abstract}

Mathematics Subject Classifications (1991): 14H05, 81R10.

Key words: separation of variables, Langlands-Drinfeld correspondence, Radon transform.

\section{Introduction}

The geometric Langlands conjectures, as formulated by Beilinson and Drinfeld in [2], aim at the construction of certain $\mathcal{D}$-modules on the moduli space of $G$ bundles over (punctured) curves ( $G$ a reductive group). Positive characteristic versions of these conjectures had been solved earlier by Drinfeld in [3], in the case $G=G L_{2}$.

In the paper [6], the $\mathcal{D}$-modules arising from the construction of [2] were studied in the special case of a rational curve with marked points, and identified with the Gaudin model. Then, in [8], Drinfeld's construction of local systems on the moduli space of rank two vector bundles on a curve in positive characteristic ([3]) was adapted to the complex situation. The identification of these two constructions amounts to Sklyanin's separation of variables ([11]), as it was noticed in [8]. This computation is recalled in the first part of this text.

The question has been raised in [8] to construct a similar separation of variables for the Gaudin-Calogero systems, which were computed in [4] and [10], and play a similar role in the case of a punctured elliptic curve. This note aims at solving this question. In the present case Drinfeld's diagrams for Radon transformation have to be slightly modified.

It is also worth to note that the systems presented here, are the specialization at the critical level, of the Knizhnik-Zamolodchikov-Bernard Equations on the torus. According to the general viewpoint that the diagrams of [3] are related to the Drinfeld-Sokolov reduction, the generalisation of the present work to noncritical level should relate these equations to the Virasoro correlators on the torus. In [5], the 
Bethe Equations were connected with the unitarity property of the KZB Equation on the torus. It would be desirable to clarify further the connection between these issues. Finally, among related works, let us quote the paper [9], where the separation of variables for classical elliptic Calogero systems was established.

\section{Separation of variables for $\mathrm{sl}_{2}$ Gaudin systems}

Let us begin with some reminders on the Gaudin system. Let $X=\mathbf{C} P^{1}, z_{\alpha}$ be marked points on $X, \alpha=1, \ldots, N, G$ be $\mathrm{SL}_{2}(\mathbf{C}), B \subset G$ be the upper triangular subgroup. The moduli space $\mathcal{M}_{G}\left(X, z_{\alpha}\right)$ of $G$-bundles on $\mathbf{C} P^{1}$, with parabolic structures at $z_{\alpha}$, is the disjoint union of the $\mathcal{M}_{G}^{(n)}\left(X, z_{\alpha}\right), n \geqslant 0$, corresponding to the parabolic structures on the sheaf $\mathcal{O}(n \infty) \oplus \mathcal{O}(-n \infty)$. We then identify $\mathcal{M}_{G}^{(n)}\left(X, z_{\alpha}\right)$ with

$$
\begin{aligned}
& P_{n} \backslash(G / B)^{N}, P_{0}=G, \quad \text { and } \\
& P_{n}=\left\{\left(\begin{array}{cc}
t & p(z) \\
0 & t^{-1}
\end{array}\right), t \in \mathbf{C}^{\times}, p(z) \in \mathbf{C}[z], \operatorname{deg}(p) \leqslant 2 n\right\}
\end{aligned}
$$

for $n>0, P_{0}$ acting diagonally and $P_{n}$ acting on the $\alpha$-th factor by left translation, after the replacement of $z$ by $z_{\alpha}$. In what follows we will deal with $\mathcal{M}_{G}^{(0)}\left(X, z_{\alpha}\right)$.

Let us fix weights, $\lambda_{\alpha}, \alpha=1, \ldots, N$. On $\mathcal{M}_{G}^{(0)}\left(X, z_{\alpha}\right)$ lives the bundle $\mathcal{L}_{\left(\lambda_{\alpha}\right)}$, quotient of the bundle $\otimes_{\alpha=1}^{N} \mathcal{L}_{\lambda_{\alpha}}\left(\mathcal{L}_{\lambda_{\alpha}}\right.$ is the line bundle on $G / B$, corresponding to the weight $\left.\lambda_{\alpha}\right)$. The natural action of $Z\left(U_{-2} \hat{\mathrm{sl}}_{2}\right)_{l o c}$ (the center of the local completion of the enveloping algebra of the central extension of $\operatorname{sl}_{2}(\mathbf{C}((z)))$ at level -2 ) is by differential operators $L_{\alpha}, \alpha=1, \ldots, N$, which were identified in [6] with the Gaudin hamiltonians

$$
L_{\alpha}=\sum_{\beta \neq \alpha} \frac{I_{a}^{(\alpha)} I^{a(\beta)}}{z_{\alpha}-z_{\beta}},
$$

$I_{a}, I^{a}$ being an orthogonal basis of $\operatorname{sl}_{2}(\mathbf{C})$.

Following the conjectures of [2], the $\mathcal{D}$-modules on $\mathcal{M}_{G}\left(X, z_{\alpha}\right)$ (twisted by $\left.\mathcal{L}_{\left(\lambda_{\alpha}\right)}\right)$ defined by $L_{\alpha}-\mu_{\alpha}$ should satisfy the Hecke eigenvalue property. In [3], a construction of such modules was given in the case of a curve of genus $>0$ without punctures.

Following [8], let us show how Drinfeld's construction in [3] can be adapted for $X=\mathbf{C} P^{1}$ with marked points $z_{\alpha}$. Consider the space

$$
\begin{aligned}
\mathcal{M}_{B}^{(0)}\left(X, z_{\alpha}\right)=\{ & \left(\text { parabolic structure on } \mathcal{O}_{\mathbf{C} P^{1}}^{2} \text { at } z_{\alpha},\right. \\
& \left.\left.\quad \text { class of morphisms } \mathcal{O}_{\mathbf{C} P^{1}} \rightarrow \mathcal{O}_{\mathbf{C} P^{1}}^{2}\right)\right\},
\end{aligned}
$$

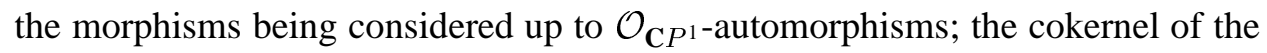
morphism considered in this definition is $\mathcal{O}_{\mathbf{C P}}$. It is natural to consider $K_{\mathbf{C} P^{1}, z_{\alpha}}=$ 
$K_{\mathbf{C} P^{1}}\left(\Sigma_{\alpha=1}^{N}\left(z_{\alpha}\right)\right)$ as the canonical bundle in our punctured situation, and then the space $\left\{\right.$ classes of morphisms $K_{\mathbf{C} P^{1}, z_{\alpha}}^{-1} \rightarrow \mathcal{O}_{\mathbf{C} P^{1}}$ \}, its mapping $\pi$ to $X^{N-2}$ (given by the zeroes of a given section) and the diagram

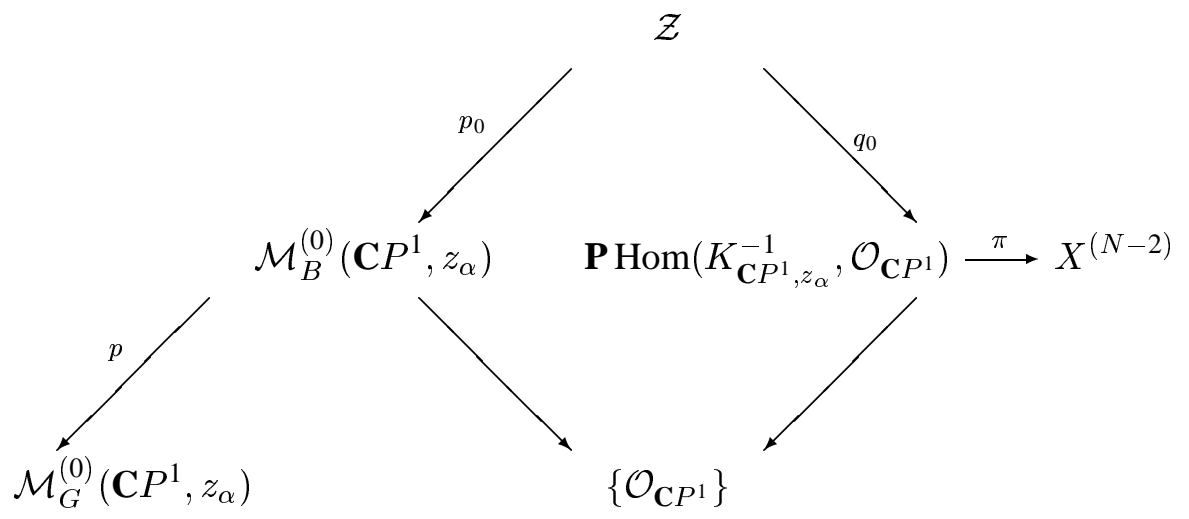

$p$ being the projection on the first factor, and the correspondence $\mathcal{Z}$ being defined to be the set of $\left(\left(l_{\alpha}, i\right), j\right), l_{\alpha}$ : line in the fiber of $\mathcal{O}_{\mathbf{C} P^{1}}^{2}$ at $z_{\alpha}$, $i$ : morphism $\mathcal{O}_{\mathbf{C} P^{1}} \rightarrow \mathcal{O}_{\mathbf{C} P^{1}}^{2}, j$ : morphism $K_{\mathbf{C} P^{1}, z_{\alpha}} \rightarrow \mathcal{O}_{\mathbf{C} P^{1}}$, proportional $i, j$ 's being considered equivalent, such that denoting by $k: \mathcal{O}_{\mathbf{C} P^{1}}^{2} \rightarrow \mathcal{O}_{\mathbf{C} P^{1}}$, the cokernel mapping of $i$, there exists a lift $j^{\prime}: K_{\mathbf{C} P^{1}, z_{\alpha}} \rightarrow \mathcal{O}_{\mathbf{C} P^{1}}^{2}$ of $j$ (i.e., we have $j=k \circ j$ ), compatible with the parabolic structure (i.e., the image of $j^{\prime}$ at $z_{\alpha}$ should be the line $l_{\alpha}$ ).

Let us fix weights $\lambda_{\alpha}$ for each $\alpha$; the $\mathcal{D}$-modules we will consider will be twisted by the following line bundles: $\mathcal{L}_{\left(\lambda_{\alpha}\right)}$ on $\mathcal{M}_{G}^{(0)}\left(X, z_{\alpha}\right), p^{*} \mathcal{L}_{\left(\lambda_{\alpha}\right)}$ on $\mathcal{M}_{B}^{(0)}\left(X, z_{\alpha}\right)$, and $p_{0}^{*} p^{*} \mathcal{L}_{\left(\lambda_{\alpha}\right)}$ on $\mathcal{Z}$. For $Y$ a variety and $\mathcal{L}$ a line bundle on $Y$, we denote $\left(\mathcal{D}_{Y}\right)_{\mathcal{L}}=\mathcal{L} \otimes$ $\mathcal{D}_{Y} \otimes \mathcal{L}^{-1}$. Let us fix now complex numbers $\mu_{\alpha}, \alpha=1, \ldots, N$, s.t. $\Sigma_{\alpha=1}^{N} \mu_{\alpha}=0$, $\Sigma_{\alpha=1}^{N} \mu_{\alpha} z_{\alpha}+\Sigma_{\alpha=1}^{N} 2 \lambda_{\alpha}\left(\lambda_{\alpha}-1\right)=0, \Sigma_{\alpha=1}^{N} \mu_{\alpha} z_{\alpha}^{2}+\Sigma_{\alpha=1}^{N} 4 \lambda_{\alpha}\left(\lambda_{\alpha}-1\right) z_{\alpha}=0$; we associate to them the operator on $X$,

$$
D_{\left(\lambda_{\alpha}\right),\left(\mu_{\alpha}\right)}=2 \partial_{w}^{2}-\sum_{\alpha=1}^{N} \frac{\mu_{\alpha}}{w-z_{\alpha}}-\sum_{\alpha=1}^{N} \frac{2 \lambda_{\alpha}\left(\lambda_{\alpha}-1\right)}{\left(w-z_{\alpha}\right)^{2}}
$$

and the $\mathcal{D}_{X}$-module $\mathcal{E}_{\left(\lambda_{\alpha}\right),\left(\mu_{\alpha}\right)}=\mathcal{D}_{X} / \mathcal{D}_{X} D_{\left(\lambda_{\alpha}\right),\left(\mu_{\alpha}\right)}$.

Consider on the other hand on $\mathcal{M}_{G}^{(0)}\left(X, z_{\alpha}\right)$ the twisted $\mathcal{D}$-module

$$
\mathcal{M}_{\left(\mu_{\alpha}\right)}=\left(\mathcal{D}_{\mathcal{M}_{G}^{(0)}\left(X, z_{\alpha}\right)}\right)_{\mathcal{L}_{\left(\lambda_{\alpha}\right)}} / \sum_{\alpha=1}^{N}\left(\mathcal{D}_{\mathcal{M}_{G}^{(0)}\left(X, z_{\alpha}\right)}\right)_{\mathcal{L}_{\left(\lambda_{\alpha}\right)}}\left(L_{\alpha}-\mu_{\alpha}\right),
$$

(the conditions on $\mu_{\alpha}$ correspond to the relations on the $L_{\alpha}$,

$$
\begin{aligned}
& \sum_{\alpha=1}^{N} L_{\alpha}=0, \quad \sum_{\alpha=1}^{N} L_{\alpha} z_{\alpha}+\sum_{\alpha=1}^{N} 2 \lambda_{\alpha}\left(\lambda_{\alpha}-1\right)=e f+f e+\frac{1}{2} h^{2}, \\
& \left.\sum_{\alpha=1}^{N} L_{\alpha} z_{\alpha}^{2}+\sum_{\alpha=1}^{N} 4 \lambda_{\alpha}\left(\lambda_{\alpha}-1\right) z_{\alpha}=2\left(e_{1} f+f_{1} e+\frac{1}{2} h_{1} h\right)\right),
\end{aligned}
$$


$e=\Sigma_{\alpha=1}^{N} e^{(\alpha)}, e_{1}=\Sigma_{\alpha=1}^{N} z_{\alpha} e^{(\alpha)}$, analogous relations for $\left.f, f_{1}, h, h_{1}\right)$. We would like to show:

PROPOSITION 1 (cf. [8].). There is a homomorphism of $\mathcal{D}$-modules

$$
\pi^{*} \mathcal{E}_{\left(\lambda_{\alpha}\right),\left(\mu_{\alpha}\right)}^{(N-2)} \rightarrow R\left(q_{0}\right)_{*} p_{1}^{*} p^{*} \mathcal{M}_{\left(\mu_{\alpha}\right)}[N],
$$

which is an isomorphism over $\pi^{-1}\left((X-\{\infty\})^{(N-2)}-\Delta\right)(\Delta$ is the diagonal part of $\left.(X-\{\infty\})^{(N-2)}\right)$.

(Here we denote, for $\mathcal{F}$ a sheaf on a manifold $V$, by $\mathcal{F}^{(n)}$ the sheaf $\left(p_{V}\right)_{*}\left(\mathcal{F}^{\square n}\right)$ on $V^{(n)}=V^{n} / S_{n}, p_{V}$ being the projection $V^{n} \rightarrow V^{(n)}$.)

Proof. Let us give coordinates to the spaces of diagram (1).

$$
\mathcal{M}_{G}^{(0)}\left(X, z_{\alpha}\right) \simeq G \backslash(G / B)^{N}=G \backslash\left(\mathbf{C} P^{1}\right)^{N},
$$

choosing the identification

$$
\mathbf{C} P^{1} \simeq G / B, \quad t \mapsto\left(\begin{array}{cc}
1 & 0 \\
t^{-1} & 1
\end{array}\right) B, \quad 0 \mapsto\left(\begin{array}{cc}
0 & -1 \\
1 & 0
\end{array}\right) B
$$

then $G$ acts on $\left(\mathbf{C} P^{1}\right)^{N}$ by homographic transformations. Now $\mathcal{M}_{B}^{(0)}\left(X, z_{\alpha}\right)=$ $B \backslash(G / B)^{N}$; after fixing $\mathcal{O}_{\mathbf{C} P^{1}} \rightarrow \mathcal{O}_{\mathbf{C} P^{1}}^{2}$ to be $(1,0)$, the lines $l_{\alpha}$ are $\mathbf{C}\left(1, t_{\alpha}^{-1}\right)$, the $t_{\alpha}^{-1}$ being defined up to a global affine transformation. An element of $\operatorname{Hom}\left(K_{\mathbf{C} P^{1}, z_{\alpha}}^{-1}\right.$, $\left.\mathcal{O}_{\mathbf{C} P^{1}}\right)$ is a 1-form $\Sigma_{\alpha=1}^{N}\left(u_{\alpha} d z / z-z_{\alpha}\right)$, with $\Sigma_{\alpha=1}^{N} u_{\alpha}=0$. The incidence relation defining $\mathcal{Z}$ is $\Sigma_{\alpha=1}^{N} u_{\alpha} t_{\alpha}=0$, since the first component of $j^{\prime}$ has to be $\Sigma_{\alpha=1}^{N}\left(u_{\alpha} t_{\alpha} d z / z-z_{\alpha}\right)$, and should be regular at $\infty$. The map $\pi$ associates to $\left(u_{\alpha}\right)$, the solutions $\left(w_{i}\right)$ of $\Sigma_{\alpha=1}^{N}\left(u_{\alpha} / z-z_{\alpha}\right)=0$ (counting $k$ times $\infty$, if this function is $\sim c / z^{2+k}$ for $z \rightarrow \infty, c \neq 0$ ).

Let $p_{1}$ be the natural projection of $(G / B)^{N}$ on $G \backslash(G / B)^{N}$, then

$$
p_{1}^{*}\left(\mathcal{M}_{\left(\mu_{\alpha}\right)}\right)=\mathcal{D}_{(G / B)^{N}} / \sum_{\alpha} \mathcal{D}_{(G / B)^{N}}\left(L_{\alpha}-\mu_{\alpha}\right)+\mathcal{D}_{(G / B)^{N}} \operatorname{sl}_{2}(\mathbf{C}) .
$$

Introduce the formal variable $z$, then

$$
\begin{aligned}
& \sum_{\alpha=1}^{N} \frac{L_{\alpha}-\mu_{\alpha}}{z-z_{\alpha}}+\sum_{\alpha=1}^{N} \frac{2 \lambda_{\alpha}\left(\lambda_{\alpha}-1\right)}{\left(z-z_{\alpha}\right)^{2}} \\
& \quad=e(z) f(z)+f(z) e(z)+\frac{1}{2} h(z)^{2}-\sum_{\alpha=1}^{N} \frac{\mu_{\alpha}}{z-z_{\alpha}}
\end{aligned}
$$


with $e(z)=\Sigma_{\alpha=1}^{N}\left(e^{(\alpha)} / z-z_{\alpha}\right)$, etc., $e^{(\alpha)}=t_{\alpha}^{2}\left(\partial / \partial t_{\alpha}\right)+2 \lambda_{\alpha} t_{\alpha}, f^{(\alpha)}=$ $-\left(\partial / \partial t_{\alpha}\right), h^{(\alpha)}=2\left(t_{\alpha}\left(\partial / \partial t_{\alpha}\right)+\lambda_{\alpha}\right)$. The Radon transform of the $\mathcal{D}$-module generated by the $L_{\alpha}-\mu_{\alpha}$ is the $\mathcal{D}$-module generated by the $\bar{L}_{\alpha}-\mu_{\alpha}$, where

$$
\begin{aligned}
& \sum_{\alpha=1}^{N} \frac{\bar{L}_{\alpha}-\mu_{\alpha}}{z-z_{\alpha}}+\sum_{\alpha=1}^{N} \frac{2 \lambda_{\alpha}\left(\lambda_{\alpha}-1\right)}{\left(z-z_{\alpha}\right)^{2}} \\
& \quad=\bar{e}(z) \bar{f}(z)+\bar{f}(z) \bar{e}(z)+\frac{1}{2} \bar{h}(z)^{2}-\sum_{\alpha=1}^{N} \frac{\mu_{\alpha}}{z-z_{\alpha}},
\end{aligned}
$$

$\bar{e}(z)=\Sigma_{\alpha=1}^{N}\left(\bar{e}^{(\alpha)} / z-z_{\alpha}\right)$, analogous formulae for $\bar{f}(z), \bar{h}(z), \bar{e}^{(\alpha)}=$ $-\left(u_{\alpha}\left(\partial / \partial u_{\alpha}\right)^{2}+2\left(\lambda_{\alpha}+1\right) \partial / \partial u_{\alpha}\right), \bar{f}^{(\alpha)}=u_{\alpha}, \bar{h}^{(\alpha)}=-2\left(u_{\alpha}\left(\partial / \partial u_{\alpha}\right)+\lambda_{\alpha}+1\right)$.

Consider the operator

$$
\hat{L}\left(w_{i}\right)=\sum_{\alpha=1}^{N} \frac{1}{w_{i}-z_{\alpha}}\left(\bar{L}_{\alpha}-\mu_{\alpha}\right)
$$

and let $\hat{e}\left(w_{i}\right)=\Sigma_{\alpha=1}^{N}\left(1 / w_{i}-z_{\alpha}\right) \bar{e}^{(\alpha)}$, analogous formulae for $\hat{f}\left(w_{i}\right), \hat{h}\left(w_{i}\right)$. Then

$$
\begin{aligned}
& \hat{L}\left(w_{i}\right)+\sum_{\alpha=1}^{N} \frac{\mu_{\alpha}}{w_{i}-z_{\alpha}}+\sum_{\alpha=1}^{N} \frac{2 \lambda_{\alpha}\left(\lambda_{\alpha}-1\right)}{\left(w_{i}-z_{\alpha}\right)^{2}} \\
& -\left[\hat{e}\left(w_{i}\right) \hat{f}\left(w_{i}\right)+\hat{f}\left(w_{i}\right) \hat{e}\left(w_{i}\right)+\frac{1}{2} \hat{h}\left(w_{i}\right)^{2}\right] \\
& =-\sum_{1 \leqslant \alpha, \beta \leqslant N} \frac{1}{w_{i}-z_{\alpha}}\left\{\left[\bar{e}^{(\alpha)}, \frac{1}{w_{i}-z_{\beta}}\right] \bar{f}^{(\beta)}+\left[\bar{f}^{(\alpha)}, \frac{1}{w_{i}-z_{\beta}}\right] \bar{e}^{(\beta)}\right. \\
& \left.+\frac{1}{2}\left[\bar{h}^{(\alpha)}, \frac{1}{w_{i}-z_{\beta}}\right] \bar{h}^{(\beta)}\right\} \\
& =-\sum_{1 \leqslant \alpha, \beta \leqslant N}-\frac{1}{w_{i}-z_{\alpha}}\left[u_{\alpha} \frac{\partial}{\partial u_{\alpha}}, \frac{\partial}{\partial u_{\alpha}}\left(\frac{1}{w_{i}-z_{\beta}}\right)\right]_{+} \cdot u_{\beta} \\
& +\frac{2}{w_{i}-z_{\alpha}} u_{\alpha} \frac{\partial}{\partial u_{\alpha}}\left(\frac{1}{w_{i}-z_{\beta}}\right)\left(u_{\beta} \frac{\partial}{\partial u_{\beta}}\right) \\
& -\sum_{1 \leqslant \alpha, \beta \leqslant N}-\frac{1}{w_{i}-z_{\alpha}}\left\{\left[2\left(\lambda_{\alpha}+1\right) \frac{\partial}{\partial u_{\alpha}}, \frac{1}{w_{i}-z_{\beta}}\right] u_{\beta}\right. \\
& \left.-\frac{2}{w_{i}-z_{\alpha}} u_{\alpha} \frac{\partial}{\partial u_{\alpha}}\left(\frac{1}{w_{i}-z_{\beta}}\right)\left(\lambda_{\beta}+1\right)\right\}
\end{aligned}
$$


with $[a, b]_{+}=a b+b a$. Now,

$$
\begin{aligned}
& \sum_{\beta=1}^{N} \frac{\partial}{\partial u_{\alpha}}\left(\frac{1}{w_{i}-z_{\beta}}\right) u_{\beta}=-\frac{1}{w_{i}-z_{\alpha}}, \\
& \sum_{\beta=1}^{N} u_{\alpha}\left(\frac{\partial}{\partial u_{\alpha}}\right)^{2}\left(\frac{1}{w_{i}-z_{\beta}}\right) u_{\beta}=-2 u_{\alpha} \frac{\partial}{\partial u_{\alpha}}\left(\frac{1}{w_{i}-z_{\alpha}}\right),
\end{aligned}
$$

so the last line gives zero, and the term in $[,]_{+}$gives

$$
\begin{aligned}
& -2 \sum_{\alpha=1}^{N} \frac{1}{w_{i}-z_{\alpha}} u_{\alpha} \frac{\partial}{\partial u_{\alpha}} \cdot \frac{1}{w_{i}-u_{\alpha}}+2 \sum_{\alpha=1}^{N} \frac{1}{w_{i}-z_{\alpha}} u_{\alpha} \frac{\partial}{\partial u_{\alpha}}\left(\frac{1}{w_{i}-u_{\alpha}}\right) \\
& =-2 \sum_{\alpha=1}^{N} \frac{1}{\left(w_{i}-z_{\alpha}\right)^{2}} u_{\alpha} \frac{\partial}{\partial u_{\alpha}} .
\end{aligned}
$$

(the dot denotes the product of differential operators). Then we deduce from

$$
C \frac{\prod_{i=1}^{N-1}\left(z-w_{i}\right)}{\prod_{\alpha=1}^{N}\left(z-z_{\alpha}\right)}=\sum_{\alpha=1}^{N} \frac{u_{\alpha}}{z-z_{\alpha}}
$$

$\left(\mathrm{d} C / C+\Sigma_{i=1}^{N-2}\left(\mathrm{~d} w_{i} / w_{i}-z\right)\right) \Sigma_{\alpha=1}^{N}\left(u_{\alpha} / z-z_{\alpha}\right)=\Sigma_{\alpha=1}^{N}\left(\mathrm{~d} u_{\alpha} / z-z_{\alpha}\right)$, so $\mathrm{d} u_{\alpha}=$ $u_{\alpha}\left(\mathrm{d} C / C+\Sigma_{i=1}^{N-2}\left(\mathrm{~d} w_{i} / w_{i}-z_{\alpha}\right)\right)$ and

$$
\frac{\partial}{\partial w_{i}}=\sum_{\alpha=1}^{N} \frac{u_{\alpha}}{w_{i}-z_{\alpha}} \frac{\partial}{\partial u_{\alpha}}
$$

so the remaining term gives $-2 \Sigma_{\beta=1}^{N}\left(\partial / \partial w_{i}\right)\left(1 / w_{i}-z_{\beta}\right)\left(u_{\beta}\left(\partial / \partial u_{\beta}\right)\right) \Sigma_{\beta=1}^{N}\left(2 /\left(w_{i}-\right.\right.$ $\left.\left.z_{\beta}\right)^{2}\right)\left(u_{\beta}\left(\partial / \partial u_{\beta}\right)\right)$.

Finally,

$$
\begin{array}{r}
\hat{L}\left(w_{i}\right)=\hat{e}\left(w_{i}\right) \hat{f}\left(w_{i}\right)+\hat{f}\left(w_{i}\right) \hat{e}\left(w_{i}\right)+\frac{1}{2} \hat{h}\left(w_{i}\right)^{2} \\
-\sum_{\alpha=1}^{N} \frac{\mu_{\alpha}}{w_{i}-z_{\alpha}}-\sum_{\alpha=1}^{N} \frac{2 \lambda_{\alpha}\left(\lambda_{\alpha}-1\right)}{\left(w_{i}-z_{\alpha}\right)^{2}} .
\end{array}
$$

Now, $\hat{f}\left(w_{i}\right)=0$, and $\hat{h}\left(w_{i}\right)=-2\left[\partial / \partial w_{i}+A\left(w_{i}\right)\right]$, with $A\left(w_{i}\right)=\Sigma_{\alpha=1}^{N}\left(\lambda_{\alpha}+\right.$ 1) $/\left(w_{i}-z_{\alpha}\right)$, so

$$
\hat{L}\left(w_{i}\right)=2\left(\frac{\partial}{\partial w_{i}}+A\left(w_{i}\right)\right)^{2}-\sum_{\alpha=1}^{N} \frac{\mu_{\alpha}}{w_{i}-z_{\alpha}}-\sum_{\alpha=1}^{N} \frac{2 \lambda_{\alpha}\left(\lambda_{\alpha}-1\right)}{\left(w_{i}-z_{\alpha}\right)^{2}} .
$$


In this way, we have constructed an epimorphism from the $(N-2)$ th symmetric power of $\mathcal{E}_{\left(\lambda_{\alpha}\right),\left(\mu_{\alpha}\right)}$ (restricted to the complement of diagonals) to the $\mathcal{D}$-module generated by the $\bar{L}_{\alpha}-\mu_{\alpha}$ 's (restricted to the complement of the discriminant), and so to the $\mathcal{D}$-module generated by the $\bar{L}_{\alpha}-\mu_{\alpha}$ 's and the action of $\operatorname{sl}_{2}(\mathbf{C})$.

Let us now show that it induces an isomorphism of the sheaves of local analytic solutions. Let us start with a local homomorphism of the first sheaf to the (analytic) structure sheaf. It is some function $\left(\psi\left(w_{i}\right)\right)_{1 \leqslant i \leqslant N-2}$, such that $\left(\partial_{w_{j}}^{2}+q\left(w_{j}\right)\right) \psi\left(w_{i}\right)=0$, for all $j$. We deduce from that relation, using our previous computations,

$$
\sum_{i=1}^{N} \frac{\left(\bar{L}_{\alpha}-\mu_{\alpha}\right) \psi}{w_{i}-z_{\alpha}}=0, \quad i=1, \ldots, N-2 .
$$

We will consider $\psi$ as a distribution on the space of all $\left(u_{i}\right)_{1 \leqslant i \leqslant N}$, supported on the hyperplane $\Sigma_{i=1}^{N} u_{i}=0$, and analytic on this hyperplane. We obtain from (3)

$$
\left(\bar{L}_{\alpha}-\mu_{\alpha}\right) \psi=u_{\alpha} \phi+z_{\alpha} u_{\alpha} \rho,
$$

$\phi$ and $\rho$ being distributions of the same nature as $\psi$; indeed, $\phi$ and $\rho$ can be obtained solving a Cramer system (since everywhere on the support of $\psi$, we can find two indices $\alpha \neq \beta$ such that $u_{\alpha} u_{\beta} \neq 0$ ), and $\bar{L}_{\alpha}$ 's commute with $\Sigma_{\alpha=1}^{N} u_{\alpha}$. From the relation $\Sigma_{\alpha=1}^{N} \bar{L}_{\alpha}=0$ follows that $\rho=0$; from $\Sigma_{\alpha=1}^{N} \bar{L}_{\alpha} z_{\alpha}+$ $\Sigma_{\alpha=1}^{N} 2 \lambda_{\alpha}\left(\lambda_{\alpha}-1\right)=\bar{e} \bar{f}+\bar{f} \bar{e}+\frac{1}{2} \bar{h}^{2}$ and $\Sigma_{\alpha=1}^{N} \bar{L}_{\alpha} z_{\alpha}^{2}+\Sigma_{\alpha=1}^{N} 4 \lambda_{\alpha}\left(\lambda_{\alpha}-1\right) z_{\alpha}=$ $2\left(\bar{e}_{1} \bar{f}+\bar{f}_{1} \bar{e}+\frac{1}{2} \bar{h}_{1} \bar{h}\right)$, follows that $\left(\Sigma_{\alpha=1}^{N} u_{\alpha}\right) \bar{e} \psi=\left(\Sigma_{\alpha=1}^{N} z_{\alpha} u_{\alpha}\right) \phi$, and $\left(\Sigma_{\alpha=1}^{N} z_{\alpha} u_{\alpha}\right) \bar{e} \psi=\left(\Sigma_{\alpha=1}^{N} z_{\alpha}^{2} u_{\alpha}\right) \phi$. So, on the complement of $\left\{\left(u_{\alpha}\right) \mid \Sigma_{\alpha=1}^{N} z_{\alpha} u_{\alpha}=0\right.$ or $\Sigma_{\alpha=1}^{N} z_{\alpha}^{2} u_{\alpha}=0$ or $\left.\left(\Sigma_{\alpha=1}^{N} z_{\alpha} u_{\alpha}\right)^{2}-\left(\Sigma_{\alpha=1}^{N} u_{\alpha}\right)\left(\Sigma_{\alpha=1}^{N} z_{\alpha}^{2} u_{\alpha}\right)\right\}, \phi$ and $\bar{e} \psi$ will vanish. So these distributions would have to be supported on a subvariety of the set of all $\left(u_{\alpha}\right)$ of codimension $\geqslant 2$, which is impossible. So we will have

$$
\left(\bar{L}_{\alpha}-\mu_{\alpha}\right) \psi=0, \quad \bar{e} \psi=0 .
$$

Since $\bar{f} \psi=\bar{h} \psi=0$ by construction, we have shown that $\psi$ can be considered as a local homomorphism of the $\mathcal{D}$-module generated by the $\bar{L}_{\alpha}-\mu_{\alpha}$ (restricted to the complement of the discriminant) to the structure sheaf.

These two morphisms are clearly inverse to each other; if we show that both $\mathcal{D}$-modules have their characteristic varieties supported on the zero section, this will prove the proposition.

For $\mathcal{E}_{\left(\lambda_{\alpha}\right),\left(\mu_{\alpha}\right)}^{(N-2)}$ it is clear, since it is true for $\mathcal{E}_{\left(\lambda_{\alpha}\right),\left(\mu_{\alpha}\right)}$. On the other hand, the characteristic variety of $R\left(q_{0}\right)_{*} p_{1}^{*} p^{*} \mathcal{M}_{\left(\mu_{\alpha}\right)}[N]$ is the set of $\left(u_{i}, \xi_{i}\right)$, with $\Sigma_{i=1}^{N} u_{i}=$ 0 and up to equivalence $\left(u_{i}, \xi_{i}\right) \sim\left(u_{i}, \xi_{i}+\lambda\right)$, and

$$
\sum_{i=1}^{N} \frac{u_{i}}{z-z_{i}}=\left(\sum_{i=1}^{N} \frac{u_{i} \xi_{i}}{z-z_{i}}\right)\left(\sum_{i=1}^{N} \frac{u_{i} \xi_{i}^{2}}{z-z_{i}}\right) .
$$


This equation gives

$$
\begin{aligned}
& \sum_{i=1}^{N} \frac{u_{i}}{z-z_{i}}=\frac{R A^{2}(z)}{\prod_{i=1}^{N}\left(z-z_{i}\right)}, \quad \sum_{i=1}^{N} \frac{u_{i} \xi_{i}}{z-z_{i}}=\frac{R A B(z)}{\prod_{i=1}^{N}\left(z-z_{i}\right)} \\
& \sum_{i=1}^{N} \frac{u_{i} \xi_{i}^{2}}{z-z_{i}}=\frac{R B^{2}(z)}{\prod_{i=1}^{N}\left(z-z_{i}\right)},
\end{aligned}
$$

$R, A, B$ polynomials. The set $\left\{w_{i}, 1 \leqslant i \leqslant N-2\right\}$ is the union of the set of zeros of $R A^{2}$, and of $\infty$ counted $N-2-\operatorname{deg} R A^{2}$ times. Since the $w_{i}$ 's are pairwise distinct, we have $A=$ const., and $\operatorname{deg} R=N-2$ or $N-3$. Since no $w_{i}$ coincides with $\infty, \operatorname{deg} R=N-2$; so, $B$ is also constant, and the $\xi_{i}$ 's are all equal; but this is equivalent to $\xi_{i}=0$.

\section{Separation of variables for the $\mathrm{sl}_{2}$ Gaudin-Calogero system}

Let $X$ be the elliptic curve $\mathbf{C}^{\times} / q^{\mathbf{Z}}$, with marked points $z_{\alpha} q^{\mathbf{Z}}, \alpha=1, \ldots, N$. In the $\mathrm{Sl}_{2}$ case, the Gaudin-Calogero system (which plays the role of the Gaudin system in the present situation, cf. [4], [10]) takes place in the space

$$
\begin{aligned}
\mathcal{M}_{G}^{(0)}\left(X, z_{\alpha}\right)= & \left\{\left(\mathcal{E}_{\left(t, t^{-1}\right)}, \text { parabolic structure at } z_{\alpha} \text { given by } t_{\alpha} \in \mathbf{C} P^{1}\right)\right\} / \\
& \left.\left.\left(t, t_{\alpha}\right) \sim\left(q t, z_{\alpha} t_{\alpha}\right),\left(t, t_{\alpha}\right) \sim\left(t, u t_{\alpha}\right), u \in \mathbf{C}^{\times}\right], t \in \mathbf{C}^{\times}\right] \\
= & \mathbf{C}^{\times} \times\left(\mathbf{C} P^{1}\right)^{N} / \mathbf{C}^{\times} \ltimes \mathbf{Z}^{N}
\end{aligned}
$$

Here $\mathcal{E}_{\left(t_{1}, \ldots, t_{n}\right)}$ is the bundle on $X$ defined by $\mathbf{C}^{\times} \times \mathbf{C}^{n} /\left[(z, \xi) \sim\left(q z, \operatorname{diag}\left(t_{i}\right) \xi\right)\right]$, for $t_{1}, \ldots, t_{n} \in \mathbf{C}^{\times}$.

We consider then the space

$$
\begin{aligned}
\mathcal{M}_{B}^{(0)}\left(X, z_{\alpha}\right) & \\
= & \left\{\left(\mathcal{E}_{\left(t, t^{-1}\right)}, j: \mathcal{E}_{t} \rightarrow \mathcal{E}_{\left(t, t^{-1}\right)}, \text { par. str. given by } t_{\alpha} \in \mathbf{C} P^{1}\right), t \in \mathbf{C}^{\times}\right\} / \\
& {\left[j \sim \lambda j, \lambda \in \mathbf{C}^{\times},\left(t, t_{\alpha}\right) \sim\left(q t, z_{\alpha} t_{\alpha}\right),\left(t, t_{\alpha}\right) \sim\left(t, u t_{\alpha}\right), u \in \mathbf{C}^{\times}\right] ; }
\end{aligned}
$$


it has a natural projection $p$ to $\mathcal{M}_{G}^{(0)}\left(X, z_{\alpha}\right)$. Consider now the diagram

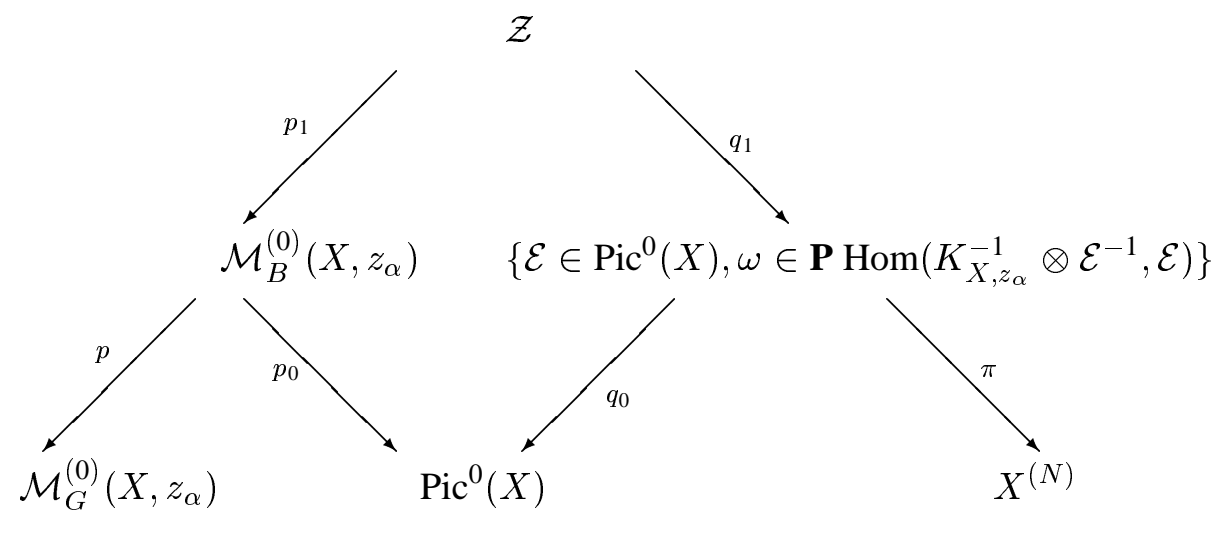

where

$$
K_{X, z_{\alpha}}=\Omega_{X}^{1}\left(\sum_{\alpha=1}^{N}\left(z_{\alpha}\right)\right)
$$

$p_{0}$ is the projection

$$
\operatorname{class}\left(\mathcal{E}_{\left(t, t^{-1}\right)}, j: \mathcal{E}_{t} \rightarrow \mathcal{E}_{\left(t, t^{-1}\right)}, \text { par. str. }\right) \mapsto \mathcal{E}_{t^{-1}}
$$

( $p_{0}$ associates to $j$ its cokernel), $q_{0}$ associates $\mathcal{E}$ to $(\mathcal{E}, \omega), \pi$ associates to $(\mathcal{E}, \omega)$ the set of zeros of $\omega$, and $\mathcal{Z}$ is the incidence variety, defined by the conditions that $\omega$ lifts to a morphism $j^{\prime}: K_{X, z_{\alpha}}^{-1} \otimes \mathcal{E}_{t^{-1}} \rightarrow \mathcal{E}_{\left(t, t^{-1}\right)}$, compatible with the parabolic structure. Writing

$$
\omega=\sum_{\alpha=1}^{N} u_{\alpha} \frac{\theta\left(t^{-2} z z_{\alpha}^{-1}\right)}{\theta\left(t^{-2}\right) \theta\left(z z_{\alpha}^{-1}\right)} \frac{\mathrm{d} z}{z}
$$

the first component of $j^{\prime}$ has to be

$$
\sum_{\alpha=1}^{N} u_{\alpha} t_{\alpha} \frac{\dot{\theta}\left(z z_{\alpha}^{-1}\right)}{\theta\left(z z_{\alpha}^{-1}\right)} \frac{d z}{z}
$$

so that the incidence condition is $\Sigma_{\alpha=1}^{N} u_{\alpha} t_{\alpha}=0$.

(Fix our conventions for $\theta$ - and $\wp$-functions: $\theta(z)=\Pi_{i \geqslant 0}\left(1-q^{i} z\right) \Pi_{i>0}(1-$ $\left.q^{i} z^{-1}\right), \wp(\ln z)=-(\dot{\theta} / \theta)(z)$, so $\wp(\zeta) \sim \zeta^{-2}+\cdots$ for $\zeta \rightarrow 0$; we denote $\dot{f}(z)=$ $z(\mathrm{~d} f / \mathrm{d} z)$.) 
The lifts to $\mathbf{C}^{\times} \times\left(\mathbf{C} P^{1}\right)^{N}$ of the Gaudin-Calogero operators ([4], [10]) are defined as follows: let $z$ be a formal variable, belonging to $X-\left\{z_{\alpha}\right\}$. We have

$$
\begin{aligned}
L(z)= & e(z) f(z)+f(z) e(z)+\frac{1}{2} h(z)^{2} \\
= & L_{0}+\sum_{\alpha=1}^{N} L_{\alpha} \frac{\dot{\theta}}{\theta}\left(z z_{\alpha}^{-1}\right)+\sum_{\alpha=1}^{N} 2 \lambda_{\alpha}\left(\lambda_{\alpha}-1\right) \wp\left(\ln z z_{\alpha}^{-1}\right) \\
& +\sum_{\alpha=1}^{N} \frac{1}{2} h^{(\alpha)}\left(\sum_{\alpha=1}^{N} h^{(\alpha)}\right)\left(\frac{\dot{\theta}}{\theta}\left(z z_{\alpha}^{-1}\right)\right)^{2}
\end{aligned}
$$

where $e(z)=\Sigma_{\alpha=1}^{N}\left(\theta\left(t^{-2} z z_{\alpha}^{-1}\right) / \theta\left(t^{-2}\right) \theta\left(z z_{\alpha}^{-1}\right)\right) e^{(\alpha)}, h(z)=2 t^{2}\left(\partial / \partial t^{2}\right)+$ $2 k(\dot{\theta} / \theta)\left(t^{2}\right)+\Sigma_{\alpha=1}^{N}(\dot{\theta} / \theta)\left(z z_{\alpha}^{-1}\right) h^{(\alpha)}, f(z)=\Sigma_{\alpha=1}^{N}\left(\theta\left(t^{2} z z_{\alpha}^{-1}\right) / \theta\left(t^{2}\right) \theta\left(z z_{\alpha}^{-1}\right)\right) f^{(\alpha)}$, and $e^{(\alpha)}=t_{\alpha}^{2}\left(\partial / \partial t_{\alpha}\right)+2 \lambda_{\alpha} t_{\alpha}, f^{(\alpha)}=-\left(\partial / \partial t_{\alpha}\right), h^{(\alpha)}=2\left(t_{\alpha}\left(\partial / \partial t_{\alpha}\right)+\lambda_{\alpha}\right)$.

Let us fix now complex numbers $\mu_{\alpha}, \alpha=0, \ldots, N$, with $\Sigma_{\alpha=1}^{N} \mu_{\alpha}=0$ (this condition corresponds to the fact that $\Sigma_{\alpha=1}^{N} L_{\alpha}$ belongs to the left ideal generated by $\left.\Sigma_{\alpha=1}^{N} h^{(\alpha)}\right)$; consider on $\mathcal{M}_{G}^{(0)}\left(X, z_{\alpha}\right)$, the $\mathcal{D}$-module (twisted by the quotient $\mathcal{L}_{k,\left(\lambda_{\alpha}\right)}$ of $i^{-1}\left(\mathcal{L}_{k}^{\bigotimes 2}\right) \otimes \nabla_{\alpha=1}^{N} \mathcal{L}_{\lambda_{\alpha}}, i: \operatorname{Ker}(s) \rightarrow X^{(2)}, s: X^{(2)} \rightarrow X$ the sum mapping, $\mathcal{L}_{k}$ a bundle of degree $k$ on $X$ ),

$$
\mathcal{M}_{\left(\mu_{\alpha}\right)}=\left(\mathcal{D}_{\mathcal{M}_{G}^{(0)}\left(X, z_{\alpha}\right)}\right) \mathcal{L}_{k,\left(\lambda_{\alpha}\right)} / \sum_{\alpha=0}^{N}\left(\mathcal{D}_{\mathcal{M}_{G}^{(0)}\left(X, z_{\alpha}\right)}\right)_{\mathcal{L}_{k,\left(\lambda_{\alpha}\right)}}\left(L_{\alpha}-\mu_{\alpha}\right)
$$

Consider then the operator on $X-\left\{z_{\alpha}\right\}$,

$$
\begin{aligned}
D_{\left(\lambda_{\alpha}\right),\left(\mu_{\alpha}\right)}= & 2\left(w \frac{\partial}{\partial w}\right)^{2}-\mu_{0}-\sum_{\alpha=1}^{N} \mu_{\alpha} \frac{\dot{\theta}}{\theta}\left(w z_{\alpha}^{-1}\right) \\
& +2 \sum_{\alpha=1}^{N} \lambda_{\alpha}\left(\lambda_{\alpha}-1\right) \wp\left(\ln w z_{\alpha}^{-1}\right)
\end{aligned}
$$

and the $\mathcal{D}_{X}$-module

$$
\mathcal{E}_{\left(\lambda_{\alpha}\right),\left(\mu_{\alpha}\right)}=\mathcal{D}_{X} / \mathcal{D}_{X} D_{\left(\lambda_{\alpha}\right),\left(\mu_{\alpha}\right)}
$$

The fibration $\pi$ has fibers $\mathbf{C}^{\times}$; we will twist inverse images under $\pi$ by the function $C^{\Sigma_{\alpha=1}^{N}\left(\lambda_{\alpha}+1\right)}(C$ coordinate on the fiber). Also, we will work with $k=0$ (multiplication by $\theta\left(t^{2}\right)^{k}$ taking us back to this case). We will show that:

PROPOSITION. There is a homomorphism of $\mathcal{D}$-modules from the twisted inverse image $\pi^{*} \mathcal{E}_{\left(\lambda_{\alpha}\right),\left(\mu_{\alpha}\right)}^{(N)}$ to $R\left(q_{1}\right)_{*} p_{1}^{*} p^{*} \mathcal{M}_{\left(\mu_{\alpha}\right)}[N]$, which is an isomorphism over $\pi^{-1}$ $\left(X^{(N)}-\Delta\right)\left(\Delta\right.$ is the diagonal part of $\left.X^{(N)}\right)$. 
Proof. Let

$$
p_{1}: \mathbf{C}^{\times} \times\left(\mathbf{C} P^{1}\right)^{N} \rightarrow \mathbf{C}^{\times} \times\left(\mathbf{C} P^{1}\right)^{N} / \mathbf{C}^{\times} \ltimes \mathbf{z}^{N}
$$

be the natural projection, then

$$
\begin{aligned}
p_{1}^{*}\left(\mathcal{M}_{\left(\mu_{\alpha}\right)}\right)= & \mathcal{D}_{\mathbf{C}^{\times} \times\left(\mathbf{C} P^{1}\right)^{N}} / \sum_{\alpha} \mathcal{D}_{\mathbf{C}^{\times} \times\left(\mathbf{C} P^{1}\right)^{N}}\left(L_{\alpha}-\mu_{\alpha}\right) \\
& +\mathcal{D}_{\mathbf{C}^{\times} \times\left(\mathbf{C} P^{1}\right)^{N}}\left(\sum_{\alpha} h^{(\alpha)}\right) .
\end{aligned}
$$

Because of the factor $\mathcal{D}_{\mathbf{C}^{\times} \times\left(\mathbf{C} P^{1}\right)^{N}}\left(\Sigma_{\alpha} h^{(\alpha)}\right)$, the $\mathcal{D}$-module is constant along the fibers of the action of $\mathbf{C}^{\times}$. Its Radon transform is the $\mathcal{D}$-module generated by $\bar{L}_{\alpha}-\mu_{\alpha}$ and $\Sigma_{\alpha=1}^{N} \bar{h}^{(\alpha)}$, where

$$
\begin{aligned}
\bar{L}_{0} & +\sum_{\alpha=1}^{N} \bar{L}_{\alpha} \frac{\dot{\theta}}{\theta}\left(z z_{\alpha}^{-1}\right)+\sum_{\alpha=1}^{N} 2 \lambda_{\alpha}\left(\lambda_{\alpha}-1\right) \wp\left(\ln z z_{\alpha}^{-1}\right) \\
& +\sum_{\alpha=1}^{N} \frac{1}{2} \bar{h}^{(\alpha)}\left(\sum_{\alpha=1}^{N} \bar{h}^{(\alpha)}\right)\left(\frac{\dot{\theta}}{\theta}\left(z z_{\alpha}^{-1}\right)\right)^{2} \\
& =\bar{e}(z) \bar{f}(z)+\bar{f}(z) \bar{e}(z)+\frac{1}{2} \bar{h}(z)^{2},
\end{aligned}
$$

$\bar{e}(z)=\Sigma_{\alpha=1}^{N}\left(\theta\left(t^{-2} z z_{\alpha}^{-1}\right) / \theta\left(t^{-2}\right) \theta\left(z z_{\alpha}^{-1}\right)\right) \bar{e}^{(\alpha)}$, analogous formulae for $\bar{f}(z)$ and $\bar{h}(z)$, with

$$
\begin{aligned}
\bar{e}^{(\alpha)} & =-\left[u_{\alpha}\left(\frac{\partial}{\partial u_{\alpha}}\right)^{2}+2\left(\lambda_{\alpha}+1\right) \frac{\partial}{\partial u_{\alpha}}\right], \quad \bar{f}^{(\alpha)}=u_{\alpha}, \\
\bar{h}^{(\alpha)} & =-2\left[u_{\alpha} \frac{\partial}{\partial u_{\alpha}}+\left(\lambda_{\alpha}+1\right)\right] .
\end{aligned}
$$

Consider the operator

$$
\begin{aligned}
\hat{L}\left(w_{i}\right)= & \bar{L}_{0}-\mu_{0}+\sum_{\alpha=1}^{N} \frac{\dot{\theta}}{\theta}\left(w_{i} z_{\alpha}^{-1}\right)\left(\bar{L}_{\alpha}-\mu_{\alpha}\right) \\
& +\sum_{\alpha=1}^{N}\left(\frac{\dot{\theta}}{\theta}\left(w_{i} z_{\alpha}^{-1}\right)\right)^{2} \frac{1}{2} \bar{h}^{(\alpha)}\left(\sum_{\alpha=1}^{N} \bar{h}^{(\alpha)}\right) .
\end{aligned}
$$

Let $\hat{e}\left(w_{i}\right)=\Sigma_{\alpha=1}^{N}\left(\theta\left(t^{-2} w_{i} z_{\alpha}^{-1}\right) / \theta\left(t^{-2}\right) \theta\left(w_{i} z_{\alpha}^{-1}\right)\right) \bar{e}^{(\alpha)}$, etc. Let us compute the difference

$$
\begin{gathered}
-\hat{L}\left(w_{i}\right)+\left(\hat{e}\left(w_{i}\right) \hat{f}\left(w_{i}\right)+\hat{f}\left(w_{i}\right) \hat{e}\left(w_{i}\right)+\frac{1}{2} \hat{h}\left(w_{i}\right)^{2}-\mu_{0}\right. \\
\left.-\sum_{\alpha=1}^{N} \mu_{\alpha} \frac{\dot{\theta}}{\theta}\left(w_{i} z_{\alpha}^{-1}\right)+2 \lambda_{\alpha}\left(\lambda_{\alpha}-1\right) \wp\left(\ln w_{i} z_{\alpha}^{-1}\right)\right)
\end{gathered}
$$


it is equal to

$$
\begin{aligned}
& \sum_{\alpha, \beta=1}^{N} \frac{\theta\left(t^{-2} w_{i} z_{\alpha}^{-1}\right)}{\theta\left(t^{-2}\right) \theta\left(w_{i} z_{\alpha}^{-1}\right)}\left[\bar{e}^{(\alpha)}, \frac{\theta\left(t^{2} w_{i} z_{\beta}^{-1}\right)}{\theta\left(t^{2}\right) \theta\left(w_{i} z_{\beta}^{-1}\right)}\right] \bar{f}^{(\beta)} \\
& +\frac{1}{2} \sum_{\alpha, \beta=1}^{N} \frac{\dot{\theta}}{\theta}\left(w_{i} z_{\alpha}^{-1}\right)\left[\bar{h}^{(\alpha)}, \frac{\dot{\theta}}{\theta}\left(w_{i} z_{\beta}^{-1}\right)\right] \bar{h}^{(\beta)} .
\end{aligned}
$$

The first term of (8) is the sum of (9) and (10), where

$$
\begin{aligned}
(9)=- & \sum_{\alpha, \beta=1}^{N} \frac{\theta\left(t^{-2} w_{i} z_{\alpha}^{-1}\right)}{\theta\left(t^{-2}\right) \theta\left(w_{i} z_{\alpha}^{-1}\right)}\left[2 u_{\alpha} \frac{\partial}{\partial u_{\alpha}}\left(\frac{\theta\left(t^{2} w_{i} z_{\beta}^{-1}\right)}{\theta\left(t^{2}\right) \theta\left(w_{i} z_{\beta}^{-1}\right)}\right) \frac{\partial}{\partial u_{\alpha}}\right. \\
& \left.+u_{\alpha}\left(\frac{\partial}{\partial u_{\alpha}}\right)^{2}\left(\frac{\theta\left(t^{2} w_{i} z_{\beta}^{-1}\right)}{\theta\left(t^{2}\right) \theta\left(w_{i} z_{\beta}^{-1}\right)}\right)\right] \cdot u_{\beta} \\
=- & \sum_{\alpha=1}^{N} \frac{\theta\left(t^{-2} w_{i} z_{\alpha}^{-1}\right)}{\theta\left(t^{-2}\right) \theta\left(w_{i} z_{\alpha}^{-1}\right)} 2 u_{\alpha}\left[\sum_{\beta=1}^{N} \frac{\partial}{\partial u_{\alpha}}\left(\frac{\theta\left(t^{2} w_{i} z_{\beta}^{-1}\right)}{\theta\left(t^{2}\right) \theta\left(w_{i} z_{\beta}^{-1}\right)}\right) u_{\beta}\right] \frac{\partial}{\partial u_{\alpha}} \\
- & \sum_{\alpha=1}^{N} \frac{\theta\left(t^{-2} w_{i} z_{\alpha}^{-1}\right)}{\theta\left(t^{-2}\right) \theta\left(w_{i} z_{\alpha}^{-1}\right)} 2 u_{\alpha} \frac{\partial}{\partial u_{\alpha}}\left(\frac{\theta\left(t^{2} w_{i} z_{\beta}^{-1}\right)}{\theta\left(t^{2}\right) \theta\left(w_{i} z_{\beta}^{-1}\right)}\right) \frac{\partial}{\partial u_{\alpha}} \\
- & \sum_{\alpha=1}^{N} \frac{\theta\left(t^{-2} w_{i} z_{\alpha}^{-1}\right)}{\theta\left(t^{-2}\right) \theta\left(w_{i} z_{\alpha}^{-1}\right)} u_{\alpha} \sum_{\beta=1}^{N}\left(\frac{\partial}{\partial u_{\alpha}}\right)^{2}\left(\frac{\theta\left(t^{2} w_{i} z_{\beta}^{-1}\right)}{\theta\left(t^{2}\right) \theta\left(w_{i} z_{\beta}^{-1}\right)}\right) u_{\beta}
\end{aligned}
$$

and

$$
\begin{aligned}
(10) & =\sum_{\alpha, \beta=1}^{N} \frac{\theta\left(t^{-2} w_{i} z_{\alpha}^{-1}\right)}{\theta\left(t^{-2}\right) \theta\left(w_{i} z_{\alpha}^{-1}\right)}(-2)\left(\lambda_{\alpha}+1\right) \frac{\partial}{\partial u_{\alpha}}\left(\frac{\theta\left(t^{2} w_{i} z_{\beta}^{-1}\right)}{\theta\left(t^{2}\right) \theta\left(w_{i} z_{\beta}^{-1}\right)}\right) u_{\beta} \\
& =\sum_{\alpha=1}^{N} 2\left(\lambda_{\alpha}+1\right) \frac{\theta\left(t^{-2} w_{i} z_{\alpha}^{-1}\right)}{\theta\left(t^{-2}\right) \theta\left(w_{i} z_{\alpha}^{-1}\right)} \frac{\theta\left(t^{2} w_{i} z_{\beta}^{-1}\right)}{\theta\left(t^{2}\right) \theta\left(w_{i} z_{\beta}^{-1}\right)} \\
& =\sum_{\alpha=1}^{N} 2\left(\lambda_{\alpha}+1\right)\left[\wp\left(\ln t^{2}\right)-\wp\left(\ln w_{i} z_{\alpha}^{-1}\right)\right] .
\end{aligned}
$$

We have

$$
\sum_{\beta=1}^{N} \frac{\partial}{\partial u_{\alpha}}\left(\frac{\theta\left(t^{2} w_{i} z_{\beta}^{-1}\right)}{\theta\left(t^{2}\right) \theta\left(w_{i} z_{\beta}^{-1}\right)}\right) u_{\beta}=-\frac{\theta\left(t^{2} w_{i} z_{\alpha}^{-1}\right)}{\theta\left(t^{2}\right) \theta\left(w_{i} z_{\alpha}^{-1}\right)}
$$


and

$$
\sum_{\beta=1}^{N}\left(\frac{\partial}{\partial u_{\alpha}}\right)^{2}\left(\frac{\theta\left(t^{2} w_{i} z_{\beta}^{-1}\right)}{\theta\left(t^{2}\right) \theta\left(w_{i} z_{\beta}^{-1}\right)}\right) u_{\beta}=-2 \frac{\partial}{\partial u_{\alpha}}\left(\frac{\theta\left(t^{2} w_{i} z_{\alpha}^{-1}\right)}{\theta\left(t^{2}\right) \theta\left(w_{i} z_{\alpha}^{-1}\right)}\right)
$$

So

$$
\begin{aligned}
(9) & =\sum_{\alpha=1}^{N} 2 u_{\alpha} \frac{\theta\left(t^{2} w_{i} z_{\alpha}^{-1}\right)}{\theta\left(t^{2}\right) \theta\left(w_{i} z_{\alpha}^{-1}\right)} \frac{\theta\left(t^{-2} w_{i} z_{\alpha}^{-1}\right)}{\theta\left(t^{-2}\right) \theta\left(w_{i} z_{\alpha}^{-1}\right)} \frac{\partial}{\partial u_{\alpha}} \\
& =\sum_{\alpha=1}^{N}(-2)\left[\wp\left(\ln w_{i} z_{\alpha}^{-1}\right)-\wp\left(\ln t^{2}\right)\right] u_{\alpha} \frac{\partial}{\partial u_{\alpha}} .
\end{aligned}
$$

The second term of (8) is the sum of (11) and (12), with

$$
\text { (11) }=2 \sum_{\alpha, \beta=1}^{N} \frac{\dot{\theta}}{\theta}\left(w_{i} z_{\alpha}^{-1}\right) u_{\alpha} \frac{\partial}{\partial u_{\alpha}}\left(\frac{\dot{\theta}}{\theta}\left(w_{i} z_{\beta}^{-1}\right)\right) u_{\beta} \frac{\partial}{\partial u_{\beta}}
$$

and

$$
\begin{aligned}
(12) & =\frac{1}{2} \sum_{\alpha, \beta=1}^{N} \frac{\dot{\theta}}{\theta}\left(w_{i} z_{\alpha}^{-1}\right) 2 u_{\alpha} \frac{\partial}{\partial u_{\alpha}}\left(\frac{\dot{\theta}}{\theta}\left(w_{i} z_{\beta}^{-1}\right)\right) 2\left(\lambda_{\beta}+1\right) \\
& =\sum_{\alpha=1}^{N}\left(\lambda_{\alpha}+1\right)\left\{-2 w_{i} \frac{\partial}{\partial w_{i}}\left[\frac{\dot{\theta}}{\theta}\left(w_{i} z_{\alpha}^{-1}\right)\right]\right\} \\
& =\sum_{\alpha=1}^{N} 2\left(\lambda_{\alpha}+1\right) \wp\left(\ln \left(w_{i} z_{\alpha}^{-1}\right)\right) .
\end{aligned}
$$

To compute (11), we express the relation between the $\partial / \partial w_{i}$ and the $\partial / \partial u_{\alpha}$ : we have

$$
\sum_{\alpha=1}^{N} u_{\alpha} \frac{\theta\left(t^{2} z z_{\alpha}^{-1}\right)}{\theta\left(t^{2}\right) \theta\left(z z_{\alpha}^{-1}\right)}=C \frac{\Pi_{i=1}^{N} \theta\left(z w_{i}^{-1}\right)}{\Pi_{\alpha=1}^{N} \theta\left(z z_{\alpha}^{-1}\right)}
$$

so

$$
\begin{aligned}
& \sum_{\alpha=1}^{N} \mathrm{~d} u_{\alpha} \frac{\theta\left(t^{2} z z_{\alpha}^{-1}\right)}{\theta\left(t^{2}\right) \theta\left(z z_{\alpha}^{-1}\right)}+\frac{\mathrm{d} t}{t} u_{\alpha}\left[\frac{\dot{\theta}}{\theta}\left(t^{2} z z_{\alpha}^{-1}\right)-\frac{\dot{\theta}}{\theta}\left(t^{2}\right)\right] \frac{\theta\left(t^{2} z z_{\alpha}^{-1}\right)}{\theta\left(t^{2}\right) \theta\left(z z_{\alpha}^{-1}\right)} \\
& \quad=C \frac{\Pi_{i=1}^{N} \theta\left(z w_{i}^{-1}\right)}{\Pi_{\alpha=1}^{N} \theta\left(z z_{\alpha}^{-1}\right)}\left[\frac{\mathrm{d} C}{C}-\sum_{i=1}^{N} \frac{\mathrm{d} w_{i}}{w_{i}} \frac{\dot{\theta}}{\theta}\left(z w_{i}^{-1}\right)\right]
\end{aligned}
$$


by inspection of the pole at $z_{\alpha}$, and because of

$$
C \frac{\Pi_{i=1}^{N} \theta\left(z_{\alpha} w_{i}^{-1}\right)}{\Pi_{\beta \neq \alpha} \theta\left(z_{\alpha} z_{\beta}^{-1}\right)}=u_{\alpha}
$$

it follows that

$$
\mathrm{d} u_{\alpha}=u_{\alpha}\left[\frac{\mathrm{d} C}{C}-\sum_{i=1}^{N} \frac{\mathrm{d} w_{i}}{w_{i}} \frac{\dot{\theta}}{\theta}\left(z_{\alpha} w_{i}^{-1}\right)\right]
$$

and so

$$
w_{i} \frac{\partial}{\partial w_{i}}=-\sum_{\alpha=1}^{N} \frac{\dot{\theta}}{\theta}\left(z_{\alpha} w_{i}^{-1}\right) u_{\alpha} \frac{\partial}{\partial u_{\alpha}} .
$$

Now

$$
\begin{aligned}
(11) & =2 \sum_{\beta=1}^{N}-w_{i} \frac{\partial}{\partial w_{i}}\left(\frac{\dot{\theta}}{\theta}\left(z_{\beta} w_{i}^{-1}\right)\right) u_{\beta} \frac{\partial}{\partial u_{\beta}} \\
& =2 \sum_{\beta=1}^{N} \wp\left(\ln w_{i} z_{\beta}^{-1}\right) u_{\beta} \frac{\partial}{\partial u_{\beta}}
\end{aligned}
$$

Finally

$$
(9)+(11)=2 \wp\left(\ln t^{2}\right) \sum_{\alpha=1}^{N} u_{\alpha} \frac{\partial}{\partial u_{\alpha}}=2 \wp\left(\ln t^{2}\right) C \frac{\partial}{\partial C}
$$

and

$$
(10)+(12)=\sum_{\alpha=1}^{N} 2\left(\lambda_{\alpha}+1\right) \wp\left(\ln t^{2}\right) .
$$

The sum of these terms is $2 \wp\left(\ln t^{2}\right)\left(C(\partial / \partial C)+\Sigma_{\alpha=1}^{N}\left(\lambda_{\alpha}+1\right)\right)$; the term $C(\partial / \partial C)+\sum_{\alpha=1}^{N}\left(\lambda_{\alpha}+1\right)$ (equal to $-\frac{1}{2} \sum_{\alpha=1}^{N} \bar{h}^{(\alpha)}$ ) is set to zero in the twisted inverse image $\pi^{*} \mathcal{E}_{\left(\lambda_{\alpha}\right),\left(\mu_{\alpha}\right)}^{(N)}$. It follows that

$$
\begin{aligned}
\hat{L}\left(w_{i}\right)= & 2\left[t^{2} \frac{\partial}{\partial t^{2}}+w_{i} \frac{\partial}{\partial w_{i}}+A\left(w_{i}\right)\right]^{2}-\mu_{0}-\sum_{\alpha=1}^{N} \mu_{\alpha} \frac{\dot{\theta}}{\theta}\left(w_{i} z_{\alpha}^{-1}\right) \\
& +\sum_{\alpha=1}^{N} 2 \lambda_{\alpha}\left(\lambda_{\alpha}-1\right) \wp\left(\ln w_{i} z_{\alpha}^{-1}\right),
\end{aligned}
$$


since $\hat{e}\left(w_{i}\right)=0$, and $\hat{h}\left(w_{i}\right)=2\left[t^{2}\left(\partial / \partial t^{2}\right)+k \frac{\dot{\theta}}{\theta}\left(t^{2}\right)+w_{i}\left(\partial / \partial w_{i}\right)+A\left(w_{i}\right)\right]$, with $A\left(w_{i}\right)=-\Sigma_{\alpha=1}^{N}\left(\lambda_{\alpha}+1\right) \dot{\theta} / \theta\left(w_{i} z_{\alpha}^{-1}\right)$. The addition of the term $A\left(w_{i}\right)$ corresponds to the twisting by a $G L(1)$-connection and does not change the $P G L(2)$-oper, as in [8]. The addition of the term $t^{2} \partial / \partial\left(t^{2}\right)$ corresponds to the fact that we are working here with the variables $\left(w_{i}, t\right)$, which are linked by the relation $t^{2} \Pi_{i=1}^{N} w_{i}=$ $\Pi_{\alpha=1}^{N} z_{\alpha}\left(\bmod . q^{\mathbf{Z}}\right)$. Our statement follows as before.

Remark. As in the rational case, one may remark that the conditions on the $\mu_{\alpha}$ 's to satisfy the Bethe ansatz equations, that can be found in [7], can be translated into the condition on the projective connection defined by them, to have a single-valued solution $\psi(z)=\Pi \theta\left(z a_{i}\right) / \Pi_{\alpha=1}^{n} \theta\left(z z_{\alpha}^{-1}\right)^{\lambda_{\alpha}}$. Thanks to the Leray formulae for Radon transformation [1], one could expect the Bethe eigenvectors to be expressed in the form

$$
\begin{aligned}
\Psi\left(t_{1}, \ldots, t_{N}\right)= & \int_{\Gamma} \frac{C^{-\sum_{\alpha=1}^{N}\left(\lambda_{\alpha}+1\right)} \psi\left(w_{1}\right) \cdots \psi\left(w_{N}\right)}{\left(\sum_{\alpha=1}^{N} u_{\alpha} t_{\alpha}\right)^{k}} \\
& \cdot \sum_{\alpha=1}^{N}(-1)^{\alpha} u_{\alpha} d u_{1} \wedge \cdots \wedge d \check{u}_{\alpha} \wedge \cdots \wedge d u_{N},
\end{aligned}
$$

$1 \leqslant k-\Sigma_{\alpha=1}^{N}\left(\lambda_{\alpha}+1\right) \leqslant N-1$, the integration being on a suitable cycle in $\mathbf{C} P^{N}$. In the general case, this formula should lead to the computation of the monodromy of the Gaudin-Calogero system (by deformation of the cycle of integration). It might be interesting to express this monodromy representation directly in terms of the one of the projective connection associated to the $\mu_{\alpha}$ 's.

\section{Acknowledgement}

We express our thanks to A. Stoyanovsky and A. Varchenko for having discussed with us the content of this paper, and to $\mathrm{C}$. Sabbah for consultations on $\mathcal{D}$-modules. V.R. was supported by the INTAS grants 93-2494 and 1010-CT93-0023, and by the CNRS; he expresses his thanks to these institutions.

\section{References}

1. d'Agnolo, A. and Schapira, P.: Quantification de Leray de la dualité projective, C. R. Acad. Sci. Paris, t .139, série I (1994) 595-8.

2. Beilinson, A. A. and Drinfeld, V. G.: Quantization of Hitchin's fibration and Langlands program, Preprint.

3. Drinfeld, V. G.: Two-dimensional representations of the fundamental group of a curve over a finite field and automorphic forms on GL(2), Amer. J. Math. 105 (1983) 85-114.

4. Enriquez, B. and Rubtsov, V.: Hitchin systems, higher Gaudin operators and $r$-matrices, Math. Res. Lett. 3 (1996) 343-57 (alg-geom/9503010).

5. Falceto, F. and Gawedzki, K.: Unitarity of the Knizhnik-Zamolodchikov-Bernard connection and the Bethe Ansatz for the elliptic Hitchin system, Commun. Math. Phys. 183 (1997) 267-90 (hep-th/9604094). 
6. Feigin, B. L., Frenkel, E. V. and Reshetikhin, N.: Gaudin model, Bethe ansatz and correlation functions at the critical level, Commun. Math. Phys. 166 (1), (1995) 27-62.

7. Felder, G. and Varchenko, A.: Integral representation of solutions of the elliptic KnizhnikZamolodchikov-Bernard equations, Int. Math. Res. Notices 5 (1995) 221-33 (hep-th/9502165).

8. Frenkel, E. V.: Affine algebras, Langlands duality and Bethe ansatz, Proc. ICMP-94, 606-42, International Press (1995), (q-alg/9506003).

9. Kuznetsov, V. B., Nijhoff, F. W. and Sklyanin, E. K.: Separation of variables for the Ruijsenaars system, Preprint solv-int/9701004.

10. Nekrasov, N.: Holomorphic bundles and many-body systems, Commun. Math. Phys. 180 (1996) 587-603 (hep-th/9503157).

11. Sklyanin, E. K.: Separation of variables in the Gaudin model, J. Sov. Math. 47 (1989) 2473-88. 